Pacific Journal of Mathematic 


\section{PERTURBING EMBEDDINGS IN CODIMENSION TWO}

\section{STANLEy OCKen}

Let $f: X^{n} \rightarrow W^{n+2}$ be a fixed embedding of manifolds, assume $X$ compact, and let $g: X \rightarrow W$ be an embedding close to $f$ in the $C^{0}$ topology. In general, $g$ and $f$ will not be concordant. What small perturbation of $g$ will yield an embedding concordant to $f$ ?

In this paper, our goal is to replace $g$ by a new embedding while altering the image manifold $g(X)$ as little as possible. In case $X$ is simply connected, the problem was solved by Cappell and Shaneson as follows: if $n$ is odd, $g(X)$ is already concordant to $f[5]$. If $n$ is even and $f$ has trivial normal bundle, $g(X)$ may be replaced by its connected sum with a knot in $W$ [4]. The current paper applies previous work of the author $[9,10]$ to study the nonsimply connected case.

Recall that embeddings $f, g: X \rightarrow W$ are concordant provided there exists an embedding $H: X \times I \rightarrow W \times I$ with $H(x, 0)=(f(x), 0)$ and $H(x, 1)=(g(x), 1)$ for $x \in X$. Results will be stated for smooth manifolds and embeddings, but are true as well for piecewise linear or topological manifolds and locally flat embeddings. We assume throughout that the manifold topology of $W$ is induced by a metric which in turn induces the supremum metric $d$ on the space of embeddings of $X$ in $W$.

The first two results describe codimension two embeddings of the product of a sphere and a compact manifold.

THEOREM A. Let $f: S^{n} \times M^{k} \rightarrow W^{n+k+2}(n \geqq 2, n+k \geqq 4)$ be an embedding with trivial normal boundle. Assume that $M$ is compact, and either $W h\left(\pi_{1}(M)\right)=0$, or $\pi_{1}(M)$ is cyclic and $n+k$ is odd. Let $Y \subset S^{n} \times M$ be a tubular neighborhood of $s \times M$, where $s \in S^{n}$ is a basepoint.

Then for $g: S^{n} \times M \rightarrow W$ sufficiently close to $f$, there exists a diffeomorphism $\psi: S^{n} \times M \rightarrow S^{n} \times M$, and an embedding $h: S^{n} \times M \rightarrow W$, such that $h \psi$ is concordant to $f$, and $h$ coincides with $g$ outside $Y$.

In the above result, the phrase " $g$ sufficiently close to $f$ " means that there exists $\varepsilon>0$ such that $d(f, g)<\varepsilon$ implies the existence of $h$ with the desired properties.

In the special case $M=T^{k}$, the $k$-torus, Theorem A remains true if $n \geqq 4$ and $S^{n}$ is replaced by any simply connected manifold [ $\S 1$, Example (ii)]. 
As an important case of Theorem A, let $W=S^{n+2} \times M$. Then $f: S^{n} \times M \rightarrow S^{n+2} \times M$ is called a "parametrized knot" in $M$ provided it is homotopic to the standard inclusion; see [5, 9] for studies of parametrized knot theory.

THEOREM B. Let $f: S^{n} \times M^{k} \rightarrow S^{n+2} \times M^{k}$ be a parametrized $M$ knot, i.e., an embedding homotopic to the standard inclusion $i_{0}$. Assume $n \geqq 2, n+k \geqq 4$, and either $\pi=\pi_{1}(M)$ is cyclic, or $W h(\pi)=0$, or $n+k$ is even and $\pi$ is finite abelian of odd order. Let $Y \subset S^{n} \times M$ be a tubular neighborhood of $s \times M$, where $s \in S^{n}$ is a basepoint. Then there exist diffeomorphisms $\phi: S^{n+2} \times M \rightarrow S^{n+2} \times M$ and is: $S^{n} \times M \rightarrow S^{n} \times M$, and a parametrized $M$-knot $h$, such that $h$ 's is concordant to of, and $h$ coincides with $i_{0}$ outside $Y$.

Thus the standard inclusion may be perturbed only near a single copy of $M$ in order to produce a placement concordant to an ambient diffeomorphism of $f$.

The above two results rely strongly on the product structure of $S^{n} \times M$ in order to obtain a good hold on the normal invariants of the "complementary maps" [5,9] of the various embeddings. By applying results of [10], we obtain results for more general manifolds. Recall that two compact manifolds $Z$ and $Y$ are normally cobordant rel boundary provided there exists a simple homotopy equivalence $h: Z \rightarrow Y$, a diffeomorphism of boundaries, whose normal invariant in $[Y, G / 0]$ vanishes.

THEOREM C. Let $f: X^{n} \rightarrow W^{n+2}$ be an embedding with trivial normal bundle; assume $n \geqq 4, X$ compact, and $W h\left(\pi_{1}(X)\right)=0$. Let $Y \subset X$ be a compact codimension zero submanifold, and denote by $\bar{C}$ the closure in $X$ of $C=X-Y$. Assume that the inclusion $Y \subset X$ induces an isomorphism of fundamental groups.

Then for $g: X \rightarrow W$ an embedding sufficiently close to $f$, there exists $h: X \rightarrow W$, concordant to $f$, such that $h(X)$ contains $g(\bar{C})$, and $Z=h(X)-g(C)$ is normally cobordant rel boundary to $Y$.

It follows easily that $Z$ and $Y$ have diffeomorphic tubular neighborhoods in $W[\S 2$, Lemma 3].

To apply Theorem $\mathrm{C}$ to the perturbation problem, let $Y$ be a "small" submanifold of $X$, e.g., a tubular neighborhood of the 2skeleton. Then the image manifolds $h(X)$ and $g(X)$ intersect at least in their large common submanifold $g(C)$, and the remaining part $Z=h(X)-g(C)$ resembles closely the manifold $g(Y)$ which would have remained in the unperturbed case $h=g$. 
1. Let $\xi$ be a 2-plane bundle over a compact manifold $X$. We first recall from $[5,10]$ the theory of embeddings of $X$ in $E(\xi)$, the total space of the disc bundle of $\xi$. If $X$ has nonempty boundary, all embeddings, homotopies, concordances, etc. are relative to the boundary. Let $\stackrel{E}{E}(\xi)$ be the total space of the circle bundle of $\xi$.

A semilocal knot in $\xi$ is an embedding $f: X \rightarrow E(\xi)$ homotopic to the zero section $i_{0}$. Call $f$ and $g$ cobordant provided $f$ is concordant to $\phi g$ for some diffeomorphism $\phi:(E(\xi), \stackrel{\circ}{E}(\xi)) \rightarrow(E(\xi), \dot{E}(\xi))$, homotopic to the identity as map of pairs. The set of cobordism equivalence classes, denoted $C(X, \xi)$ or just $C(X)$, admits an abelian group structure which is defined geometrically by composition of embeddings. See $[5,10]$ for details.

To compute $C(X)$, let $\Phi$ be the commutative square

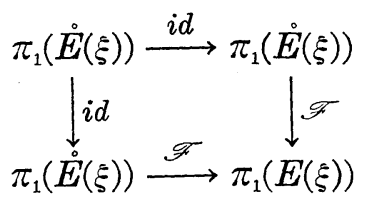

where $\mathscr{F}$ is induced by inclusion. Let $S_{X}(\xi)=S:[\Sigma E, G / 0] \rightarrow L_{n+3}(\mathscr{F})$ be the Wall surgery obstruction for the pair $(E(\xi), \stackrel{\circ}{E}(\xi))$, relative to $E(\xi \mid \partial X)$; here $n$ is the dimension of $X$. Then

Proposition [5, 5.2]. For $n \geqq 4$, there is an exact sequence

$$
0 \longrightarrow C(X) \stackrel{\Sigma}{\longrightarrow} \Gamma_{n+3}(\Phi) \stackrel{\rho}{\longrightarrow} \operatorname{cok} S \text {. }
$$

The middle term is an algebraic $K$ theoretic functor introduced in [5] for the study of homology equivalent manifolds. The homomorphism $\Sigma$ is the surgery obstruction of a normal map whose domain is the closed complement of a tubular neighborhood of an embedding, and $\rho$ is a natural homomorphism.

For $\partial X$ nonempty, the above result holds provided a semilocal knot is required to coincide with the zero section on $\partial X[10]$. This permits the study of naturality properties for codimension zero embeddings $j: Y \rightarrow X$. Let $C$ be the closure of the complement of the image of $j$; then $\partial C=\partial Y \cup \partial X$. Given a semilocal knot $f: Y \rightarrow$ $E(\xi \mid j(Y))$, define $j(f)=f \cup i_{0} \mid C: X=Y \cup C \rightarrow E(\xi \mid j(Y)) \cup E(\xi \mid C)=$ $E(\xi)$. This defines a natural homomorphism $j_{\sharp}: C(Y) \rightarrow C(X)$. Let

$$
\mathscr{F}_{X}: \pi_{1}(\stackrel{\circ}{E}(\xi)) \longrightarrow \pi_{1}(E(\xi)) \text { and } \mathscr{F}_{Y}: \pi_{1}(\stackrel{\circ}{E}(\xi \mid j(Y))) \longrightarrow \pi_{1}(E(\xi \mid j(Y)))
$$

be induced by inclusions. Then $[5,5.2]$ easily implies

LEMMA 1. If $j_{*}: \pi_{1}(Y) \rightarrow \pi_{1}(X)$ is an isomorphism, then $j_{\sharp}$ : 
$C(Y) \rightarrow C(X)$ is injective. If in addition the induced map on Wall groups satisfies $j_{*}\left(\right.$ Image $\left.S_{Y}\right)=\operatorname{Image}\left(S_{X}\right) \subset L_{n+3}\left(\mathscr{F}_{X}\right)$, then $j_{\#}$ is an isomorphism.

Here $S_{X}:[\Sigma E(\xi), G / 0] \rightarrow L_{n+3}\left(\mathscr{F}_{X}\right) \quad$ and $\quad S_{Y}:[\Sigma E(\xi \mid j(Y)), G / 0] \rightarrow$ $L_{n+3}\left(\mathscr{F}_{Y}\right)$ are the appropriate surgery obstruction maps.

In order to study perturbations of embeddings, we shall require $j_{\sharp}: C(Y) \rightarrow C(X)$ to be an epimorphism. Call $j: Y \rightarrow X$ a perfect embedding if this is the case.

Proposition 1. Assume dimension $X=n \geqq 4$ and $f: X \rightarrow E(\xi)$ is a semilocal knot. If $j: Y \rightarrow X$ is a perfect embedding, there exist

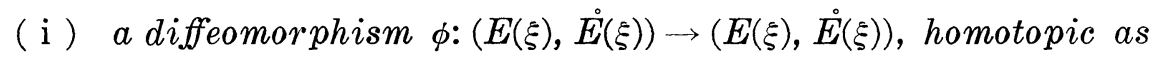
a map of pairs to the identity, and

(ii) a semilocal knot $g: X \rightarrow E(\xi)$, concordant to $\phi i_{0}$, such that $g$ and $f$ coincide on $X-Y$.

In other words, $f$ may be made cobordant to the zero section if it is allowed to be changed on $Y$.

Proof. Let $[h] \in C(Y)$ be a cobordism class for which $j_{\sharp}([h])=$ $-[f] \in C(X)$. Let $\bar{f}: E(\xi) \rightarrow E(\xi)$ be the canonical extension of $f[5$, Lemma 4.2], and set $g=\bar{f} \circ j(h)$. By definition of the group operation in $C(X),[g]=[f]+[j(h)]=\left[i_{0}\right]$. Since $j(h) \mid X-Y$ coincides with $i_{0}$, the result follows.

In order to remove the ambient diffeomorphism from the above result, it is necessary to restrict the bundle $\xi$. Let $p_{n}^{\prime}: L_{n}\left(\pi_{1}(X)\right) \rightarrow$ $L_{n+2}\left(\mathscr{F}_{X}\right)$ be defined by using the projection $p: E(\xi) \rightarrow X$ to induce a normal map to $(E(\xi), E(\xi))$ from a normal map to $X$. Call the bundle $\xi n$-perfect provided $p_{n+2}^{!}$is surjective and $p_{n+1}^{!}$is injective. The next result follows from the proof of [4, Lemma 2].

LEMMA 2. Let $\xi$ be an n-perfect 2-plane bundle over a compact manifold $X^{n}, n \geqq 4$. If a diffeomorphism $\phi:(E(\xi), \stackrel{\circ}{E}(\xi)) \rightarrow(E(\xi), \stackrel{\circ}{E}(\xi))$ is homotopic as a map of pairs to the identity, then $\phi$ is concordant to a bundle map covering a diffeomorphism $\psi: X \rightarrow X$.

THEOREM 1. Let $f: X^{n} \rightarrow W(n \geqq 4)$ be a codimension two embedding with n-perfect normal bundle $\xi$. Suppose the inclusion $Y \subset X$ of a codimension zero submanifold is a perfect embedding.

Then for any $g: X \rightarrow W$ sufficiently close to $f$, there exists a diffeomorphism $\psi: X \rightarrow X$, homotopic to the identity, and an em- 
bedding $h: X \rightarrow W$ which coincides with $g$ outside $Y$, such that $h$ is concordant to $f \psi$.

Proof. For $g$ sufficiently close to $f, g(X)$ will lie in $E(\xi)$, and $g$ and $f$ will be homotopic as maps to $E(\xi)$. Thus $g$ is a semilocal knot in $E(\xi)$, and $f$ plays the role of the zero section. Now apply the last two results.

We now give examples of bundles $\xi$ and embeddings $j: Y \rightarrow X$ which satisfy the hypotheses. The results of $[5,4]$ show that in many cases with $\pi_{1}(X)$ trivial, the conclusion of Theorem 1 holds when $Y$ is either empty or a disc.

If $\xi$ is a trivial bundle and $\pi=\pi_{1}(X)$, then $p_{n}^{\prime}$ factors as $L_{n}(\pi) \rightarrow L_{n}^{h}(\pi) \rightarrow L_{n+2}(\pi \times Z \rightarrow \pi)$. The second map is an isomorphism obtained by crossing with a disc [11]; the first is the forgetful homomorphism, which fits into an exact sequence involving $H^{*}\left(Z_{2}, W h(\pi)\right)$. If particular, $\xi$ is $n$-perfect if $H^{n+2}\left(Z_{2}, W h(\pi)\right)$ vanishes, e.g., if $W h(\pi)$ vanishes or $\pi$ is finite cyclic and $n$ is odd $[8,1]$. If $\xi$ is nontrivial but $n$ is even and $\pi_{1}(X)=0$, then $\xi$ is $n$-perfect; see [4].

We now list examples of perfect embeddings. In all cases, assume $\xi$ is trivial.

ExAmPle (i). Let $j: D^{n} \times M \rightarrow S^{n} \times M$ be the Cartesian product of $\mathrm{id}_{M}$ with an embedding of a disc in $S^{n}$. The homomorphisms $S$ for $D^{n} \times M$ and $S^{n} \times M$ in the exact sequence [5, 5.2] may be identified with the surgery obstruction maps

$$
\begin{aligned}
& \sigma:\left[\Sigma\left(S^{n} \times M\right), G / 0\right] \longrightarrow L_{n+1}^{h}\left(\pi_{1}(M)\right) \text { and } \\
& \sigma:\left[\Sigma\left(D^{n} \times M\right), G / 0\right] \longrightarrow L_{n+1}^{h}\left(\pi_{1}(M)\right),
\end{aligned}
$$

which have the same image by [9, Lemma 15.1]. Lemma 1 and the Five Lemma complete the result. This example, together with Theorem 1, implies Theorem A of the Introduction.

Example (ii). Suppose $j: T^{n} \times D^{k} \rightarrow Y$ induces a fundamental group isomorphism, $T^{n}$ the $n$-torus. If $k \geqq 4$, then $S:\left[\Sigma\left(T^{n} \times D^{k}\right)\right] \rightarrow$ $L_{n+k+1}(n Z)$ is surjective [3]. Now apply Lemma 1 .

EXAMPLE (iii). Assume $j: Y \subset X$ induces an isomorphism of finite cyclic odd order fundamental groups. Then the surgery obstruction $\sigma:[\Sigma X, G / 0] \rightarrow L_{n+1}^{h}\left(\pi_{1}(X)\right)$ is zero [3] and may be identified with $S_{X}:[\Sigma E, G / 0] \rightarrow L_{n+3}(\mathscr{F})$ provided $\xi$ is trivial. Now apply Lemma 1 to see that $j$ is a perfect embedding.

As an application of Example (i) above, we settle a question 
raised by the author's study of $G_{n}(M)$, the set of cobordism classes of "parametrized $M$-knots," i.e., embeddings $f: S^{n} \times M \rightarrow S^{n+2} \times M$ homotopic to the standard embedding $i_{0}$. Two such embeddings $f$ and $g$ are cobordant provided there exist diffeomorphisms $\phi: S^{n+2} \times M \rightarrow$ $S^{n+2} \times M$ and $\psi: S^{n} \times M \rightarrow S^{n} \times M$, each commuting up to homotopy with projection to $M$, such that $\phi f \psi$ is concordant to $g$. See $[5,9]$ for details.

Proof of Theorem $B$. We may assume that $Y$ contains the image of an embedding $j: D^{n} \times M \rightarrow S^{n} \times M$ as in Example (i) above. Consider the composite

$$
C\left(D^{n} \times M\right) \stackrel{i \sharp}{\longrightarrow} C\left(S^{n} \times M\right) \stackrel{i}{\longrightarrow} G_{n}(M) .
$$

The semilocal knot groups are those for the trivial bundle; the map $i$ is induced by the inclusion $S^{n} \times M \times D^{2} \subset S^{n+2} \times M$, and is a well defined isomorphism by [9, 15.3]. By Example (i) above and Lemma $1, j_{\sharp}$ is also an isomorphism. The stated result follows from the surjectivity of the composite. The injectivity of the composite implies that the cobordism class of a parametrized $M$-knot is determined by its behavior near a single copy of $M$ in $S^{n} \times M$.

2. In order to prove Theorem $\mathrm{C}$, we review the results of [10] on embeddings in $E(\xi)$ of manifolds simple homotopy equivalent to $X$. As before, $\xi$ is a 2-plane bundle over the compact manifold $X^{n}$.

Definition. A fake semilocal knot in $\xi$ is an embedding $f: Z \rightarrow$ $E(\xi)$, where $Z$ is a manifold of dimension $n$, such that for some simple homotopy equivalence of pairs $h:(X, \partial X) \rightarrow(Z, \partial Z)$, a diffeomorphism on the boundary, the composite $f \circ h$ is homotopic, relative to $\partial X$, to the zero section.

Two fake knots $f_{i}: Z_{i} \rightarrow E(\xi)(i=1,2)$ are cobordant provided there exist diffeomorphisms $\psi: Z_{1} \rightarrow Z_{2}$ and $\phi: E(\xi) \rightarrow E(\xi)$, the latter homotopic as a map of pairs to the identity, such that $\phi f_{2} \psi$ is concordant to $f_{1}$. Let $\hat{C}(X, \xi)$ be the set of cobordism equivalence classes.

To compute $\hat{C}(X, \xi)$, recall from $\S 1$ the natural homomorphism $j_{*}: \Gamma_{i}(\Phi) \rightarrow L_{i}(\mathscr{F})$. As before, $p^{\prime}: L_{i-2}(\pi) \rightarrow L_{i}(\mathscr{F})$ is obtained by inducing normal maps. Define

$$
\Gamma_{i}(\Phi, \xi)=j_{i}^{-1}\left(\operatorname{Image} p^{1}\right) \subset \Gamma_{i}(\Phi) .
$$

THEOREM 3. Let $\xi$ be a 2 plane bundle over a compact manifold $X^{n}(n \geqq 4)$. There is a natural surgery obstruction $\Sigma: \widehat{C}(X, \xi) \rightarrow \Gamma_{n+3}(\Phi)$ which is injective with image $\Gamma_{n+3}(\Phi, \xi)$. 
Proof. This follows from minor modifications of the proof of [10, Theorem 1]. Note that $\hat{C}(X, \xi)$ acquires an abelian group structure; this is interpreted geometrically in [10].

The following result shows that $\hat{C}(X, \xi)$ often consists of local knots which are only mildly fake. Assume the hypotheses of Theorem 3.

LEMMA 3. If $\partial X$ is nonempty, and $f: Z \rightarrow E(\xi)$ is a fake semilocal knot with tubular neighborhood $T$, then $Z$ is normally cobordant, rel boundary, to $X$, and $T$ is diffeomorphic to $E(\xi)$.

Proof. Let $h: X \rightarrow Z$ be a simple homotopy equivalence of pairs with $f \circ h$ homotopic to the zero section, and let $\bar{h}: E(\xi) \rightarrow T$ be a covering bundle map. As in [10], there is a "characteristic map" $\hat{F}: E(\xi) \rightarrow E(\xi) \bigcup_{E(\xi) \times 0}^{\circ} \stackrel{\circ}{E}(\xi) \times I \approx E(\xi)$, such that $\hat{F} \mid T=(\bar{h})^{-1}$, where $\hat{F}$ is a simple homotopy equivalence with vanishing normal invariant in $[E(\xi), G / 0]$. By naturality of normal invariants $[2,13],(\bar{h})^{-1}, \bar{h}$, and $h$ all have vanishing normal invariants. In particular, $Z$ is normally cobordant to $X$ in all cases. If $\partial X$ is nonempty, Van Kampen's theorem implies easily that the inclusion $\partial E(\xi) \subset E(\xi)$ induces a fundamental group isomorphism. By the $\pi-\pi$ theorem [13], the simple homotopy equivalence $\bar{h}$ is homotopic as a map of pairs (although not relative to $E(\xi \mid \partial X)$ ) to a diffeomorphism.

Now suppose $j: Y \rightarrow X$ is a codimension zero embedding. As in $\S 1, j$ induces a natural homomorphism $j_{\sharp}: \widehat{C}(Y, \xi \mid j(Y)) \rightarrow \widehat{C}(X, \xi)$, defined by gluing a fake semilocal knot in $\xi \mid j(Y)$ to the zero section of $\xi \mid X-Y$.

For most applications, it is necessary to assume that $p^{\prime}: L_{n+1}(\pi) \rightarrow$ $L_{n+3}(\mathscr{F})$ is surjective, where $\pi=\pi_{1}(X)$ and $\mathscr{F}$ is induced by including $\stackrel{\circ}{E}(\xi)$ in $E(\xi)$.

Proposition 2. Assume $\xi$ is a 2-plane bundle over the compact manifold $X^{n}$, and let $j: Y^{n} \rightarrow X^{n}$ induce a fundamental group isomorphism. Then $p_{X}^{!}$is surjective if and only if $p_{Y}^{!}$is surjective. In this case $j_{\sharp}: \hat{C}(Y, \xi \mid j(Y)) \rightarrow \widehat{C}(X, \xi)$ is an isomorphism.

Proof. The first assertion follows from the naturality of $p$. Theorem 3 now yields an isomorphism $\Sigma_{X}: \hat{C}(X) \rightarrow \Gamma_{n+3}\left(\Phi_{X}\right)$, where $\Phi_{x}$ is determined by $\pi_{1}(\stackrel{\circ}{E}(\xi)) \rightarrow \pi_{1}(E(\xi))$; similarly $\Sigma_{Y}: \hat{C}(Y) \rightarrow \Gamma_{n+3}\left(\Phi_{Y}\right)$ is an isomorphism. Since $j$ induces an isomorphism of the squares $\Phi_{X}$ and $\Phi_{Y}$, the result follows by the naturality of $\Sigma$. 
In order to prove Theorem $\mathrm{C}$ we combine restrictions on $p^{\text {: }}$ that occur in the above result and in $\S 1$.

Proposition 3. Let $f: X^{n} \rightarrow W(n \geqq 4)$ be a codimension two embedding with normal bundle $\xi$, and let $j: Y^{n} \rightarrow X^{n}$ induce a fundamental group isomorphism. Assume $p_{n+2}^{1}$ is surjective and $p_{n+1}^{1}$ is an isomorphism. Then for any embedding $g: X \rightarrow W$ which is sufficiently close to $f$, there exists an embedding $\theta: X \rightarrow E(\xi)$ such that

(i ) $\bar{g} \theta$ is concordant to $f$, where $\bar{g}: E(\xi) \rightarrow W$ is an embedding extending $g$

(ii) $\theta(X) \cap E(\xi \mid(X-Y))=i_{0}(X-Y)$, and

(iii) $\theta(X) \cap E(\xi \mid j(Y))$ is normally cobordant, rel boundary, to $Y$.

Proof. As in $\S 1$, assume $g(X) \subset E(\xi)$ and view $g$ as a semilocal knot in $\xi$. By Proposition 2, $j_{\sharp}: \hat{C}(Y) \rightarrow \widehat{C}(X)$ is an isomorphism; hence $\left[i_{0}\right]=[g]+j_{\sharp}[k]$ for some fake knot $k: Z \rightarrow E(\xi \mid j(Y))$. Since $g$ is a nonfake semilocal knot, addition of a fake knot to $g$ may be defined by composition; see [10] for definitions. Thus $\left[i_{0}\right]=[\bar{g} \circ j(k)]$ and there is a commutative diagram

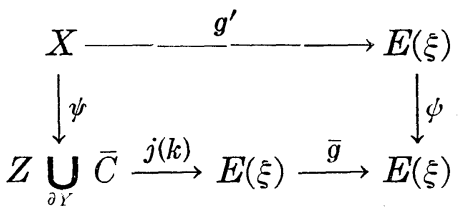

in which $\bar{C}=X$ - interior $(Y), j(k)=k \cup i_{0} \mid \bar{C}, g^{\prime}$ is concordant to $i_{0}$, and $\phi$ and $\psi$ are diffeomorphisms. By Lemma $2, \phi$ is concordant to a bundle map covering a diffeomorphism $\psi_{1}: X \rightarrow X$. Set $\theta=$ $j(k) \circ \psi \circ \psi_{1}^{-1}$; then $\bar{g} \theta$ is concordant to $i_{0}$. Properties (ii) and (iii) follow from the definition of $j(k)$ and from Lemma 3.

In order to satisfy the hypotheses, it seems necessary to restrict to $W h\left(\pi_{1}(X)\right)=0$ and $\xi$ the trivial bundle. Theorem $\mathrm{A}$ now follows by taking $h=\bar{g} \theta$.

\section{REFERENCES}

1. H. Bass, $L_{3}$ of finite abelian groups, Ann. of Math., 99 (1974), 118-153.

2. W. Browder, Surgery on Simply-Connected Manifolds, Springer Verlag, 1972.

3. W. Browder, T. Petrie, and C. T. C. Wall, The classification of free actions of cyclic groups of odd order on homotopy spheres, Bull. Amer. Math. Soc., 77 (1971), 455-459.

4. S. E. Cappell and J. L. Shaneson, Close codimension two embeddings of even dimensional manifolds, Amer. J. of Math., 97 (1975), 733-740.

5. S. E. Cappell and J. L. Shaneson, The codimension two placement problem and 
homology equivalent manifolds, Ann. of Math., 99 (1974), 278-348.

6. M. Kervaire, Les noeuds de dimension supérieures, Bull. Soc. Math. France, 93 (1965), 225-271.

7. J. Levine, Knot cobordism groups in codimension two, Comment. Math. Helvet., 44 (1968), 229-244.

8. J. W. Milnor, Whitehead torsion, Bull. Amer. Math. Soc., 72 (1966), 358-426.

9. S. Ocken, Parametrized Knot Theory, Memoirs of the AMS, 170 (1976).

10. S. Ocken, The Commutativity of Tunnel Sum, Duke Math. Journal, 43 (1976), 285-294.

11. J. L. Shaneson, Wall's surgery obstruction groups for $G \times Z$, Ann. of Math., 90 (1969), 296-334.

12. D. Sullivan, Triangulating Homotopy Equivalences, Ph. D. Thesis, Princeton, 1965.

13. C. T. C. Wall, Surgery on Compact Manifolds, Academic Press, 1970.

Received July 26, 1977. Research partially supported by a CUNY-FRAP grant.

CUNY

NEW YoRK, NY 10031 



\section{PACIFIC JOURNAL OF MATHEMATICS}

EDITORS

DONALD BABBITT (Managing Editor)

University of California

Los Angeles, California 90024

HUGo RossI

University of Utah

Salt Lake City, UT 84112

C. C. MOORE

J. DUGUNDJI

Department of Mathematics

University of Southern California

Los Angeles, California 90007

R. FinN AND J. MiLgraM

Stanford University

Stanford, California 94305

University of California

Berkeley, CA 94720

\section{ASSOCIATE EDITORS}

E. F. BrCKENBACH

B. H. NeUmanN

F. WOLF

K. YoshidA

\section{SUPPORTING INSTITUTIONS}

UNIVERSITY OF BRITISH COLUMBIA

UNIVERSITY OF SOUTHERN CALIFORNIA

CALIFORNIA INSTITUTE OF TECHNOLOGY

STANFORD UNIVERSITY

UNIVERSITY OF CALIFORNIA

UNIVERSITY OF HAWAII

MONTANA STATE UNIVERSITY

UNIVERSITY OF TOKYO

UNIVERSITY OF NEVADA, RENO

UNIVERSITY OF UTAH

NEW MEXICO STATE UNIVERSITY

WASHINGTON STATE UNIVERSITY

OREGON STATE UNIVERSITY

UNIVERSITY OF WASHINGTON

UNIVERSITY OF OREGON 


\section{Pacific Journal of Mathematics}

\section{Vol. 81, No. $1 \quad$ November, 1979}

Thomas E. Armstrong, Simplicial subdivision of infinite-dimensional compact cubes ..................................... 1

Herbert Stanley Bear, Jr., Approximate identities and pointwise convergence ................................

Richard David Bourgin, Partial orderings for integral representations on convex sets with the Radon-Nikodým property..................

Alan Day, Herbert S. Gaskill and Werner Poguntke, Distributive lattices

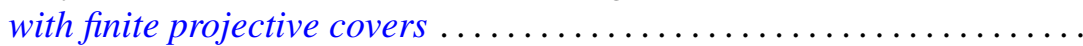

Heneri Amos Murima Dzinotyiweyi and Gerard L. G. Sleijpen, A note on measures on foundation semigroups with weakly compact orbits ......

Ronald James Evans, Resolution of sign ambiguities in Jacobi and Jacobsthal sums ...................................

John Albert Fridy, Tauberian theorems via block dominated matrices ......

Matthew Gould and Helen H. James, Automorphism groups retracting onto

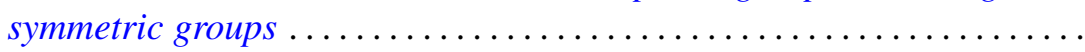

Kurt Kreith, Nonlinear differential equations with monotone solutions . . . . 101

Brian William McEnnis, Shifts on indefinite inner product spaces........ 113

Joseph B. Miles, On entire functions of infinite order with radially

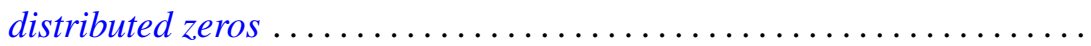

Janet E. Mills, The idempotents of a class of 0-simple inverse semigroups ...............................

Edward Jean Moulis, Jr., Generalizations of the Robertson functions ...

Richard A. Moynihan and Berthold Schweizer, Betweenness relations in probabilistic metric spaces.......................

Stanley Ocken, Perturbing embeddings in codimension two ....

Masilamani Sambandham, On the average number of real zeros of a class of random algebraic curves.

Jerry Searcy and B. Andreas Troesch, A cyclic inequality and a related eigenvalue problem.

Roger R. Smith and Joseph Dinneen Ward, $M$-ideals in $B\left(l_{p}\right)$...

Michel Talagrand, Deux généralisations d'un théorème de I. Namioka ..

Jürgen Voigt, $O n Y$-closed subspaces of $X$, for Banach spaces $X \subset Y$;

existence of alternating elements in subspaces of $C(J)$

Sidney Martin Webster, On mapping an $n$-ball into an $(n+1)$-ball in complex spaces

David J. Winter, Triangulable subalgebras of Lie p-algebras ... 